\title{
CORRIGENDUM
}

\section{The autism and schizophrenia associated gene CYFIP1 is critical for the maintenance of dendritic complexity and the stabilization of mature spines}

M Pathania, EC Davenport, J Muir, DF Sheehan, G López-Doménech and JT Kittler

Translational Psychiatry (2014) 4, e423; doi:10.1038/tp.2014.36; published online 5 August 2014

Correction to: Translational Psychiatry (2014) 4, e374; doi:10.1038/ tp.2014.16; published online 25 March 2014

While this work was under revision, a study was published showing CYFIP1 translocates between the FMRP-containing protein synthesis machinery and the actin-regulatory complex, and that disrupting CYFIP1 altered dendritic spine length, maturation and the levels of phalloidin-labeled spine F-actin. This work was incorrectly referenced (ref 34 in our manuscript) and should be cited as (De Rubeis et al., 2013). The corrected version of Reference 34 is as follows:

De Rubeis S, Pasciuto E, Li KW, Fernández E, Di Marino D, Buzzi A et al. CYFIP1 coordinates mRNA translation and cytoskeleton remodeling to ensure proper dendritic spine formation. Neuron 2013; 79: 1169-1182. 